\title{
Cálculo en agotamiento de secciones de fábrica reforzadas a flexión con láminas de FRP
}

\section{U.L.S. method for predicting flexural capacity in FRP-strengthened masonry sections}

$\underline{\text { S. Martínez }}^{(*)}$, M. D. García ${ }^{(* *)}$, J. P. Gutiérrez ${ }^{(* * *)}$

\section{RESUMEN}

Las estructuras de fábrica apenas tienen capacidad para resistir tracciones lo que las hace vulnerables ante acciones desestabilizantes de tipo accidental, como terremotos, o cuando sufren daños que alteran su geometría, por ejemplo, por asientos diferenciales.

En este trabajo se presenta un método para la comprobación en agotamiento de secciones de fábrica reforzadas a flexión con láminas de FRP. Está basado en el procedimiento de cálculo de la capacidad resistente de secciones de hormigón pero adaptado a las particularidades y condicionantes de las fábricas reforzadas. En esta propuesta se utiliza un diagrama de cálculo tensión deformación de la fábrica de tipo bilineal y se limita la deformación de cálculo del refuerzo teniendo en consideración ciertos aspectos que provocan que no pueda desarrollar toda su resistencia. En concreto, se propone un «coeficiente reductor por adherencia» que se determina a partir de una amplia base de datos con ensayos de flexión.

Palabras clave: Refuerzo; material compuesto; FRP; estructuras de fábrica; análisis numérico.

\section{ABSTRACT}

Masonry structures can hardly resist tensile loads. For this reason these structures are vulnerable to accidental actions, such as earthquakes, or when they suffer damage that change their geometry conditions, for instead, due to differential soil settlements.

This paper presents an ultimate limit state method for checking FRP-strengthened unreinforced masonry sections. The method is similar to the one used for reinforced concrete members, but it is adapted to masonry and FRP laminates particularities. In this proposal a bilinear strength-strain relationship is used and the FRP design strain is limited to take into account different aspects which cause the plate can't reach its ultimate strength. In particular, it is proposed a "bond reduction factor", derived from a large bending test database.

Keywords: Strengthening; composite material; FRP; masonry structures; numerical analysis.

(*) Dra. Arquitecta, Madrid (España).

(**) ETS de Arquitectura - Universidad Politécnica de Madrid (España).

(***) Instituto de Ciencias de la Construcción Eduardo Torroja (IETcc-CSIC), Madrid (España).

Persona de contacto/Corresponding author: soniamartinezdemingo@hotmail.com (S. Martínez)

Cómo citar este artículo/Citation: Martínez, S., García, M. D, Gutiérrez, J. P. (2015). Cálculo en agotamiento de secciones de fábrica reforzadas a flexión con láminas de FRP. Informes de la Construcción, 67(540): e118, doi: http://dx.doi.org/10.3989/ic.15.005. Licencia / License: Salvo indicación contraria, todos los contenidos de la edición electrónica de Informes de la Construcción se distribuyen bajo una licencia de uso y distribución Creative Commons Reconocimiento no Comercial 3.o. España (cc-by-nc). 


\section{INTRODUCCIÓN, ANTECEDENTES Y OBJETIVO DEL TRABAJO}

\subsection{Introducción}

Los polímeros reforzados con fibras, también conocidos como FRP, presentan unas propiedades idóneas para su empleo en el campo del refuerzo estructural tales como su elevada resistencia a tracción en el sentido longitudinal de las fibras, su bajo peso o el hecho que no sufran corrosión como el acero.

Entre otras posibles aplicaciones, se emplean en forma de láminas para el refuerzo exterior a flexión, cortante, o compresión (encamisados) (1). La mayor parte de estos refuerzos se han hecho en estructuras de hormigón armado, si bien existen experiencias con otros materiales como fábricas (Figura 1) o madera. De hecho, algunos países con importante patrimonio arquitectónico y elevado riesgo sísmico, como Italia, ya están aprovechando las propiedades de estos materiales en la rehabilitación estructural de edificios históricos (2) (3). Las estructuras de fábrica empiezan a estar presentes en algunas de las guías técnicas de diseño para refuerzos con materiales compuestos avanzados elaboradas por distintos organismos internacionales (4) (5) (6).

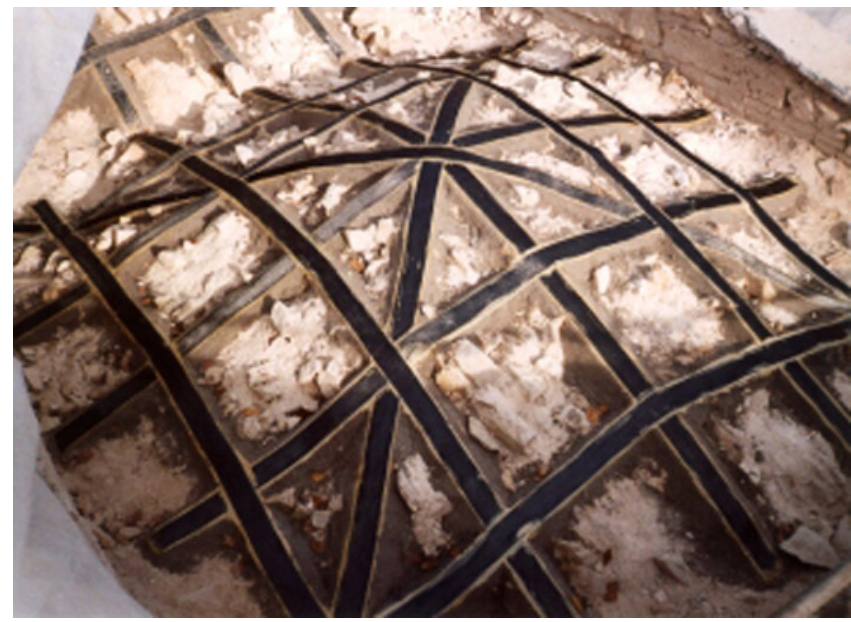

Figura 1. Solución de refuerzo con bandas preconformadas de carbono adheridas sobre las bóvedas de la Iglesia de Santa Croce en Sassoferrato, Italia (extraído de la página web de la empresa $I A R$, Ingegneria y Architecttura del Restauro: www.iar-restauri.it).

En relación con el refuerzo a flexión con materiales compuestos de fábricas, sus posibles campos de aplicación tienen que ver con situaciones donde se puedan producir solicitaciones de flexión que resulten excesivas para este material. Por ejemplo, cuando exista riesgo de que se produzcan acciones externas desestabilizantes de tipo accidental (terremotos, derrumbes parciales de otros elementos), cuando se vaya a incrementar la carga soportada por el elemento (cambios de uso, modificaciones estructurales), o en fábricas que han experimentado daños previos (asientos diferenciales, desplomes) que generan solicitaciones de flexión no previstas inicialmente.

\subsection{Antecedentes}

Se han publicado distintos trabajos experimentales en los que se ha constatado la eficacia de esta técnica aplicada al refuerzo a flexión de fábricas, si bien la mayoría son ensayos de flexión simple, situación poco habitual en fábricas reales (7) (8) (9) (10) (11) (12). Resultan de mayor interés aquellos ensayos que combinan solicitaciones de compresión y flexión (13) (14) (15) (16).

A nivel de cálculo, destaca la propuesta del profesor Triantafillou (17) quien plantea un procedimiento basado en el método de cálculo de secciones de hormigón armado y las prescripciones del EC-6. A pesar del interés de la formulación propuesta presenta la limitación de atender sólo a una de las posibles roturas: el fallo por compresión de la fábrica. En general, se observa que en los métodos de cálculo propuestos se modela la fábrica bien como un material plástico (9) (11) (12) (17) bien como un material elástico (7) (8) en función del tipo de fallo (atribuible a la fábrica o al refuerzo, respectivamente) que cada autor considere que es el que puede tener más incidencia en la práctica.

$\mathrm{Al}$ igual que en los refuerzos en hormigón, interesa evitar fallos prematuros por desprendimiento repentino de la lámina (18). El despegue iniciado en el extremo se debe evitar mediante un adecuado diseño y cálculo de la longitud de anclaje del refuerzo. Incluso, cuando la adherencia entre lámina y soporte pueda estar muy comprometida, pueden disponerse mecanismos de anclaje suplementarios. Pero también puede darse el fallo prematuro por desprendimiento de la lámina iniciado en la zona intermedia del elemento, fenómeno que suele estar ligado a la apertura de fisuras. Una forma de abordar este problema consiste en limitar la deformación (y por tanto la tensión) en el refuerzo a un valor por debajo del cual se haya probado experimentalmente que no cabe esperar fallos por despegue. Esta segunda vía facilita la práctica del proyecto del refuerzo y es la que ha seguido el procedimiento de cálculo que se presenta.

\subsection{Objetivo del trabajo}

El objetivo del trabajo realizado consiste en desarrollar un procedimiento de cálculo que permita dimensionar y/o comprobar secciones de fábrica reforzadas con materiales compuestos solicitadas a flexión y flexocompresión. En dicho procedimiento se han tenido en consideración aspectos como la dificultad de desarrollar una adherencia perfecta entre refuerzo y soporte.

\section{PROCEDIMIENTO DE CÁLCULO PROPUESTO}

El método que se propone está basado en el procedimiento que habitualmente se emplea para el cálculo en agotamiento de secciones de hormigón armado, si bien se ha adaptado a los condicionantes y particularidades de las estructuras de fábrica reforzadas con materiales compuestos avanzados.

Es de aplicación para fábricas formadas por piezas ortoédricas y aparejo regular. Está planteado para comprobar muros de sección rectangular (de ancho $b$ y espesor $t$ ) con refuerzos dispuestos en vertical (de ancho $b_{f}$ y espesor $t_{f}$ ) sometidos a esfuerzos combinados de compresión $\left(N_{d}\right)$ y flexión fuera del plano del muro $\left(M_{d}\right)$ cuyo plano de rotura sea paralelo a los tendeles.

Como se ha comentado en la introducción, en esta propuesta el despegue del refuerzo inducido por la apertura de fisuras en el interior del elemento se previene limitando su deformación de cálculo (en base a resultados de campañas experimentales). Por este motivo, las formas de fallo fundamentales de una sección de fábrica reforzada exteriormente con materiales compuestos y sometida a esfuerzos combinados de 
flexión y compresión se agrupan de la siguiente forma: 1- fallo debido a la compresión de la fábrica y 2- fallo por un exceso de deformación en la lámina de refuerzo que provoca, bien su rotura por tracción, bien su desprendimiento iniciado en la zona interior del elemento.

\subsection{Hipótesis de cálculo}

El análisis de las secciones de fábrica reforzadas en el E.L.U. de agotamiento resistente se hace a partir de las siguientes hipótesis de cálculo:

- Caracterización del E.L.U. Las situaciones de agotamiento relativas a las distintas solicitaciones normales para las fábricas reforzadas son: compresión centrada, compresión excéntrica, flexión compuesta y flexión simple.

- Compatibilidad de deformaciones en la sección. Se admite como válida la hipótesis de Bernouilli, es decir, para deformaciones pequeñas las secciones planas permanecen planas.

- Respecto a la fábrica: no se tiene en cuenta en los cálculos su resistencia a tracción por su escasa entidad y se utiliza un diagrama de cálculo tensión-deformación de tipo bilineal (Figura 2) que está en consonancia con el diagrama general propuesto por el CTE DB-SE F. Las expresiones [1] y [2] definen las dos fases del diagrama. La pendiente de la primera fase (asimilable a un valor teórico del módulo elástico de la fábrica a efectos de cálculo, $E_{m}$ ) se expresa como el producto de una constante $\beta$ por la resistencia de cálculo de la fábrica $\left(f_{m d}\right)$.

$$
\begin{aligned}
& \text { Si } 0<\varepsilon_{m} \leq \varepsilon_{m e}, \sigma=E_{m} \varepsilon_{m} \\
& \text { Si } \varepsilon_{m e}<\varepsilon_{m} \leq \varepsilon_{m u}, \sigma=f_{m d}
\end{aligned}
$$

- Respecto al material compuesto se utiliza un diagrama de cálculo tensión-deformación de tipo lineal (Figura 3) donde son de aplicación las expresiones [3] y [4]. La colaboración del refuerzo se considera plena para deformaciones inferiores a su deformación de cálculo, denominada «deformación efectiva de cálculo» $\left(\varepsilon_{f e}\right)$. Para su determinación se emplean dos coeficientes reductores aplicados sobre la deformación última del refuerzo $\left(\varepsilon_{f u}{ }^{*}\right)$. Dichos coeficientes reductores tienen en consideración dos aspectos que provocan que el material no llegue a desarrollar toda su capacidad resistente: los factores medioambientales $\left(C_{E}\right)$ y el desprendimiento del mismo inducido por la apertura de fisuras en la zona interior del elemento $(K)$. Como valores de $C_{E}$ se pueden tomar los recomendados por las guías ACI (4) (19) Tabla 1. En cuanto al valor del factor $K$, en el apartado 3.3 se presenta una propuesta para cuantificarlo. En los cálculos no se tienen en cuenta el espesor de la lámina.

$$
\begin{gathered}
f_{f d}=E_{f} \varepsilon_{f e} \\
\varepsilon_{f e}=K C_{E} \varepsilon_{f u}^{*}
\end{gathered}
$$

- Para valores de deformación del refuerzo por debajo del valor de la deformación efectiva de cálculo del refuerzo $\left(\varepsilon_{f f}\right)$ se considera que la adherencia entre refuerzo y soporte es lo suficientemente buena como para que la colaboración estructural del mismo sea plena.

- No se contemplan efectos de $2^{0}$ orden debido a la carga axial.

- La capacidad a cortante debe comprobarse a parte con la normativa técnica vigente.

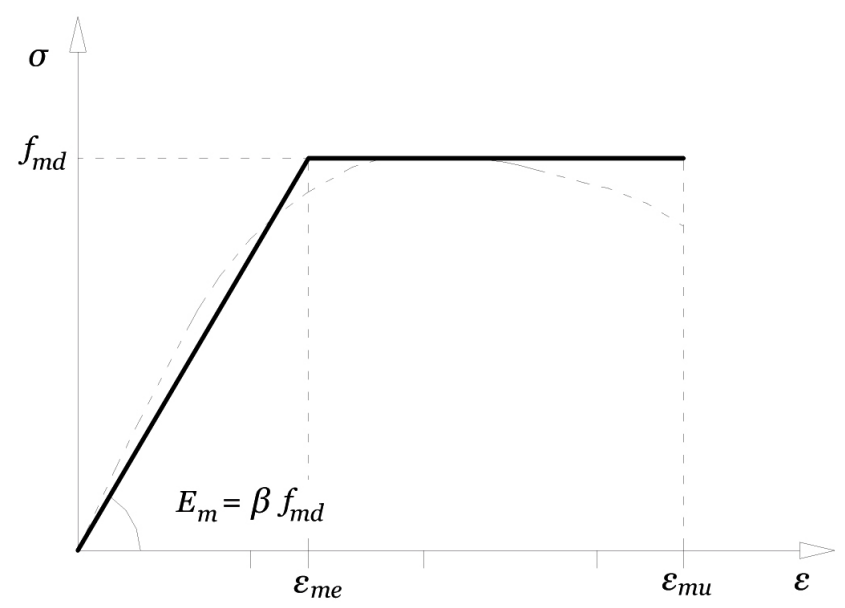

Figura 2. Diagrama de cálculo tensión-deformación de la fábrica.

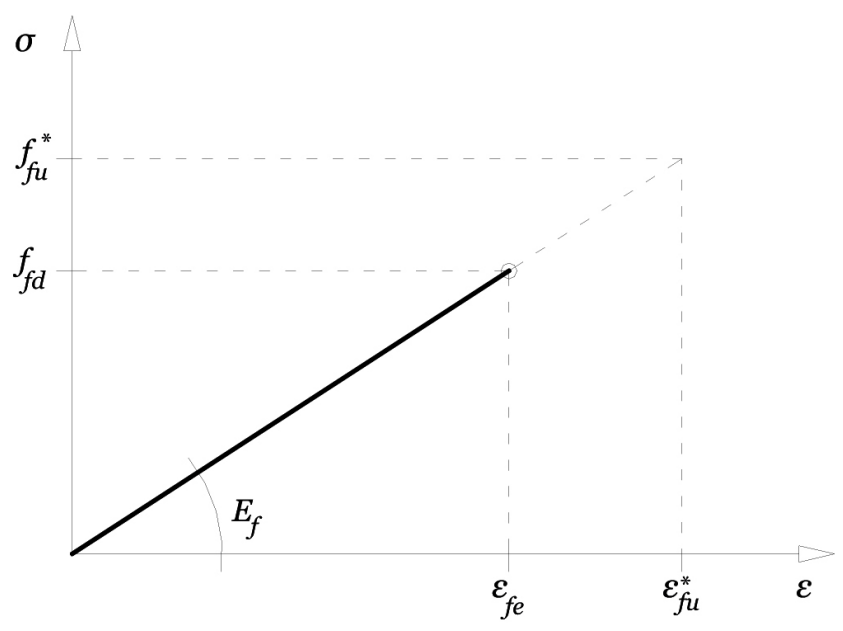

Figura 3. Diagrama de cálculo tensión-deformación del refuerzo.

Tabla 1. Coeficiente exposición medioambiental según guías técnicas del ACI.

\begin{tabular}{|c|c|c|}
\hline Tipo exposición & Tipo de fibra & $\boldsymbol{C}_{\boldsymbol{E}}$ \\
\hline \multirow{3}{*}{ Exposición interior } & Carbón & 0,95 \\
\cline { 2 - 3 } & Vidrio & 0,75 \\
\cline { 2 - 3 } & Aramida & 0,85 \\
\hline \multirow{3}{*}{ Exposición exterior } & Carbón & 0,85 \\
\cline { 2 - 3 } & Vidrio & 0,65 \\
\cline { 2 - 3 } & Aramida & 0,75 \\
\hline \multirow{3}{*}{ Ambiente agresivo } & Carbón & 0,85 \\
\cline { 2 - 3 } & Vidrio & 0,50 \\
\cline { 2 - 3 } & Aramida & 0,70 \\
\hline
\end{tabular}

\subsection{Dominios de deformación de la sección en la rotura}

El agotamiento se puede caracterizar por el valor de la deformación en determinadas fibras de la sección. Estas condiciones se agrupan de forma simplificada en los distintos dominios de deformación representados en la Figura 4.

Cada uno de estos dominios se corresponde con un tipo de rotura. El fallo de la sección reforzada bajo solicitaciones de flexión y flexocompresión puede ser debido bien a que la fábrica alcanza su deformación última a compresión (dominio B), bien a un exceso de deformaciones en el refuerzo (dominio A) donde se engloban tanto fallos por tracción de la lámina como por despegue inducido por fisuras en la parte intermedia del elemento. 

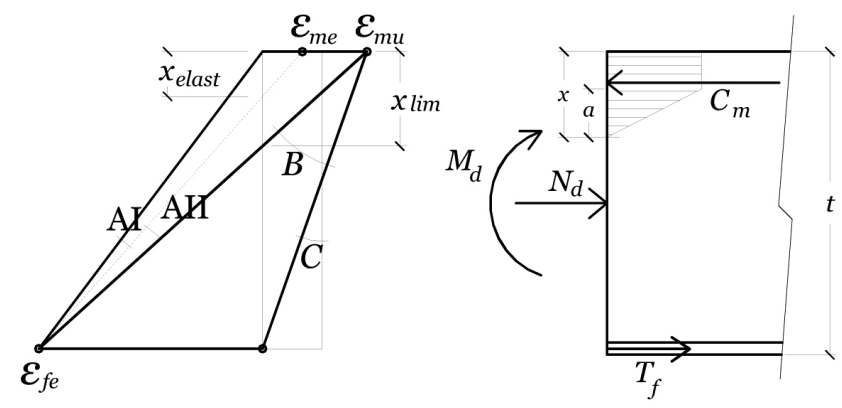

Figura 4. Dominios de deformación de la sección reforzada en la rotura.

Dentro del «domino A» se distingue una región AI, donde el refuerzo falla antes de que la fibra más comprimida de la fábrica alcance la deformación máxima del tramo elástico $\left(\varepsilon_{m e}\right)$, y una región AII donde la fábrica está ya en la región plástica. Si el dominio es de tipo C, donde predomina la solicitación de compresión, no tiene sentido proceder al refuerzo a flexión con materiales compuestos avanzados.

La cantidad de refuerzo adherido al muro puede expresarse en términos de cuantía mecánica $\left(\omega_{f}\right)$ definida como el cociente de la capacidad mecánica del refuerzo entre la capacidad mecánica de la fábrica [5].

$$
\varpi_{f}=\left(b_{f} t_{f} f_{f d}\right) /\left(b t f_{m d}\right)
$$

Planteando el equilibrio de fuerzas y momentos y la compatibilidad de deformaciones en la sección, se pueden obtener expresiones para el cálculo de las cuantías mecánicas de refuerzo que limitan estos dominios. La cuantía de refuerzo que limita los dominios AI y AII se ha denominado «cuantía mecánica elástica» $\left(\omega_{f, \text { eláss }}\right)$ y puede calcularse con la expresión [6]. La cuantía mecánica de refuerzo que limita los dominios AII y B se ha denominado «cuantía mecánica límite» $\left(\omega_{f, \text { lim }}\right)$ y puede calcularse con la expresión [7]. En ambas expresiones $v$ es el axil reducido y $\beta$ es una constante (ver Figura 2) que permite estimar de forma aproximada el módulo elástico de la fábrica a partir de su resistencia a compresión. En la bibliografía sobre fábricas el valor de esta constante suele estar comprendido entre 400 y 1000 (20).

$$
\begin{gathered}
\varpi_{f, \text { lást }}=\frac{0,5}{\left(1+\beta \mathcal{E}_{f e}\right)}-v \\
\varpi_{f, \lim }=\left(1-\frac{0,5}{\beta \varepsilon_{m u}}\right) \frac{1}{\left(1+\frac{\varepsilon_{f e}}{\varepsilon_{m u}}\right)}-v
\end{gathered}
$$

\subsection{Formulación propuesta}

A continuación se expone la formulación propuesta para cada dominio (Figura 4).

Para cuantías de refuerzo inferiores a la «cuantía mecánica elástica», el dominio de deformación es de tipo AI. En este dominio el fallo es debido al refuerzo cuya deformación es igual al valor de cálculo $\left(\varepsilon_{f f}\right)$ mientras que la deformación de la fibra más comprimida de la fábrica $\left(\varepsilon_{m}\right)$ es menor que su deformación máxima del tramo elástico $\left(\varepsilon_{m e}\right)$. Planteando las ecuaciones de equilibrio de fuerzas y momentos y la compati- bilidad de deformaciones en la sección, se obtienen las expresiones [8] y [9] con las que calcular la profundidad relativa de la fibra neutra $(x / t)$ y el momento reducido $(\mu)$.

$$
\begin{gathered}
0,5 \beta \varepsilon_{f e}\left(\frac{x}{t}\right)^{2}+\left(\omega_{f}+v\right)\left(\frac{x}{t}\right)-\left(\omega_{f}+v\right)=0 \\
\mu=0,5 \beta \varepsilon_{f e} \frac{\left(\frac{x}{t}\right)^{2}}{\left(1-\frac{x}{t}\right)}\left(0,5-\frac{1}{3} \frac{x}{t}\right)+0,5 \omega_{f}
\end{gathered}
$$

Para cuantías de refuerzo superiores a la «cuantía mecánica elástica» e inferiores a la «cuantía mecánica límite», el dominio de deformación es de tipo AII. En este caso, el fallo es también debido al refuerzo, cuya deformación es igual al valor de cálculo $\left(\varepsilon_{f e}\right)$, pero la deformación de la fibra más comprimida de la fábrica $\left(\varepsilon_{m}\right)$ es mayor que su deformación elástica $\left(\varepsilon_{m e}\right)$. Las expresiones [10], [11] y [12] permiten calcular la profundidad relativa de la fibra neutra y el momento reducido.

$$
\begin{gathered}
\left(\frac{x}{t}\right)=\left(v+\omega_{f}+0,5 \frac{1}{\beta \varepsilon_{f e}}\right) /\left(1+0,5 \frac{1}{\beta \varepsilon_{f e}}\right) \\
\mu=0,5\left(\frac{x}{t}-\frac{a}{t}\right)\left(1-\frac{x}{t}+\frac{a}{t}\right)+0,5\left(\frac{a}{t}\right)\left(0,5-\frac{x}{t}+\frac{2 a}{3 t}\right)+0,5 \omega_{f}[11] \\
\operatorname{con}\left(\frac{a}{t}\right)=\frac{\varepsilon_{m e}}{\varepsilon_{f e}}\left(1-\frac{x}{t}\right)
\end{gathered}
$$

Para cuantías mayores que la «cuantía mecánica límite» el dominio es de tipo B con fallo debido a la fábrica (la fibra más comprimida de la sección de fábrica alcanza la deformación de rotura, $\varepsilon m u$, mientras que la deformación del refuerzo es menor que su valor de cálculo). Desarrollando de nuevo las ecuaciones de equilibrio de fuerzas y momentos y la compatibilidad de deformaciones en la sección, se obtienen las expresiones correspondientes a este dominio, [13], [14] y [15].

$$
\begin{gathered}
\left(1-0,5 \frac{\varepsilon_{m e}}{\varepsilon_{m u}}\right)\left(\frac{x}{t}\right)^{2}+\left(\omega_{f} \frac{\varepsilon_{m u}}{\varepsilon_{f e}}\right)\left(\frac{x}{t}\right)-\left(\omega_{f} \frac{\varepsilon_{m u}}{\varepsilon_{f e}}\right)=0 \\
\mu=0,5\left(\frac{x}{t}-\frac{a}{t}\right)+\left(1-\frac{x}{t}+\frac{a}{t}\right)+0,5\left(\frac{a}{t}\right)\left(0,5-\frac{x}{t}+\frac{2 a}{3 t}\right)+0,5 \omega_{f} \frac{\varepsilon_{m u}}{\varepsilon_{f e}}\left(\frac{1-\frac{x}{t}}{\frac{x}{t}}\right) \\
\operatorname{con}\left(\frac{a}{t}\right)=\frac{\varepsilon_{m e}}{\varepsilon_{m u}}\left(\frac{x}{t}\right)
\end{gathered}
$$

\section{REVISIÓN DEL MÉTODO Y DETERMINACIÓN DE UN COEFICIENTE REDUCTOR POR ADHERENCIA}

\subsection{Base de datos}

Con objeto de poder comprobar la validez del procedimiento de cálculo propuesto se recopila una base de datos con 68 ensayos de flexión y flexocompresión procedentes de 9 trabajos experimentales suficientemente documentados, (7) (8) (9) (10) (11) (12) (13) (14) (17) y parte de una pequeña campaña experimental propia (16). El objetivo es doble: por un lado 
poder verificar la fiabilidad del método de cálculo propuesto con el mayor número de resultados experimentales posibles, y por otro, utilizar estos ensayos para determinar un «coeficiente reductor por adherencia» con el que estimar adecuadamente la deformación efectiva de cálculo del refuerzo $\left(\varepsilon_{f e}\right)$.

Como criterio general, sólo se incluyen en la base de datos las pruebas cuya rotura sea por flexión (tracción del refuerzo, compresión de la fábrica o despegue del laminado inducido por la fisuración intermedia). No se consideran aquellos ensayos en los que el fallo predominante es por agotamiento a cortante de la fábrica.

La base de datos la forman un total de 68 ensayos. De ellos, 56 pruebas $(82,35 \%)$ son de flexión simple y 12 (17,65\%) son de flexocompresión. Respecto al tipo de fábrica ensayada, 47 son de ladrillo $(69,12 \%)$ con predominio del ladrillo de tipo macizo (30 ensayos) frente al ladrillo perforado (17 ensayos). En los 21 ensayos restantes (30,88 \%) las probetas están formadas por bloques de hormigón. En cuanto al material empleado como refuerzo, 43 pruebas son tejidos flexibles (no preconformados) de fibra de vidrio $(63,24 \%), 12$ son tejidos flexibles de fibra de carbono (17,65\%), 7 son bandas preconformadas de carbono $(10,29 \%)$ y las 6 pruebas restantes son tejidos flexibles (no preconformados) de fibra de aramida (8,82 \%).

En la Tabla 2 se recogen los principales datos de estos ensayos: estudio del que procede, tipo de ensayo, propiedades y geometría del refuerzo, propiedades, geometría y tipo de fábrica y esbeltez de la probeta. En (16) puede encontrarse más información de las pruebas que conforman la base de datos.

Tabla 2. Principales datos de los ensayos incluidos en la base de datos.

\begin{tabular}{|c|c|c|c|c|c|c|c|c|c|c|c|c|c|c|c|c|c|}
\hline \multirow{11}{*}{\begin{tabular}{|l|}
\multicolumn{1}{|c}{ Estudio } \\
$\begin{array}{l}\text { V.-Dimas } \\
\text { (200o) }\end{array}$
\end{tabular}} & \multirow{2}{*}{$\begin{array}{c}\text { Probeta } \\
\text { S 75 } \\
\end{array}$} & $\begin{array}{l}\text { Tipo de } \\
\text { ensayo }\end{array}$ & \multicolumn{2}{|c|}{$\begin{array}{c}\text { Tipo de } \\
\text { refuerzo }\end{array}$} & \multirow{2}{*}{\begin{tabular}{|c|}
$\begin{array}{c}\boldsymbol{E}_{f} \\
(\mathbf{M P a})\end{array}$ \\
10000 \\
\end{tabular}} & \multirow{2}{*}{$\begin{array}{c}\varepsilon_{f u}{ }^{*} \\
0,020\end{array}$} & \multirow{2}{*}{$\begin{array}{l}n \\
1\end{array}$} & $\begin{array}{c}b_{f} \\
(\mathbf{m m})\end{array}$ & $\begin{array}{c}t_{f} \\
(\mathrm{~mm})\end{array}$ & Observ. & $\begin{array}{l}\text { Tipo de } \\
\text { fábrica }\end{array}$ & $\begin{array}{c}f_{\text {mk }} \\
(\mathbf{M P a})\end{array}$ & $\boldsymbol{\beta}$ & $\varepsilon_{m u}$ & $\begin{array}{c}b \\
(\mathrm{~mm})\end{array}$ & $\begin{array}{c}t \\
(\mathbf{m m})\end{array}$ & $h / t$ \\
\hline & & & GFRP & no preim & & & & 303 & 2 & & LM & \begin{tabular}{|l|}
26,7 \\
\end{tabular} & 750 & 0,003 & 1220 & 50 & 14 \\
\hline & S 25 & & GFRP & no preim & 10000 & 0,020 & 1 & 102 & 2 & & LM & 26,7 & 750 & 0,003 & 1220 & 50 & 14 \\
\hline & S20 & & GFRP & no preim & 10000 & 0,020 & 1 & 81 & 2 & & LM & 26,7 & 750 & 0,003 & 1220 & 50 & 14 \\
\hline & S 40 & & GFRP & no preim & 10000 & 0,020 & 1 & 162 & 2 & & LM & 26,7 & 750 & 0,003 & 1220 & 50 & 14 \\
\hline & S30 & flexión & GFRP & bidir. no pre & 5900 & 0,016 & 1 & 243 & 2 & refuerzo & LM & 26,7 & 750 & 0,003 & 1200 & 50 & 14 \\
\hline & $S_{100}$ & simple & GFRP & no preim & 10000 & 0,020 & 1 & 405 & 2 & anclado & LM & 26,7 & 750 & 0,003 & 1220 & 50 & 28 \\
\hline & S100 & & GFRP & no preim & 10000 & 0,020 & 1 & 405 & 2 & & LM & 26,7 & 750 & 0,003 & 1220 & 50 & 28 \\
\hline & S 200 & & GFRP & no preim & 10000 & 0,020 & 1 & 810 & 2 & & LM & 26,7 & 750 & 0,003 & 1220 & 50 & 28 \\
\hline & S50 & & GFRP & no preim & 10000 & 0,020 & 1 & 201 & 2 & & LM & 26,7 & 750 & 0,003 & 1220 & 50 & 28 \\
\hline & D 100 & & GFRP & no preim & 10000 & 0,020 & 1 & 840 & 2 & & LM & 26,7 & 750 & 0,003 & 1220 & 100 & 27 \\
\hline Morbin & $\mathrm{COG}_{3} \mathrm{R}$ & & GFRP & no preim & 72345 & 0,021 & 1 & 76,2 & 0,36 & & $\mathrm{BH}$ & 9,74 & 900 & 0,0025 & 609,6 & 101,6 & 12 \\
\hline$(2002)$ & COG5R & & GFRP & no preim & 72345 & 0,021 & 1 & 127 & 0,36 & refuerzo & $\mathrm{BH}$ & 9,74 & 900 & 0,0025 & 609,6 & 101,6 & 12 \\
\hline & CLG3R & flexión & GFRP & no preim & 72345 & 0,021 & 1 & 76,2 & 0,36 & $\sin$ & LP & 17,23 & 700 & 0,0035 & 609,6 & 101,6 & 12 \\
\hline & CLG5R & simple & GFRP & no preim & 72345 & 0,021 & 1 & 127 & 0,36 & anclar & LP & 17,23 & 700 & 0,0035 & 609,6 & 101,6 & 12 \\
\hline & CLG7R & & GFRP & no preim & 72345 & 0,021 & 1 & 177,8 & 0,36 & & LP & 17,23 & 700 & 0,0035 & 609,6 & 101,6 & 12 \\
\hline & COG5A & & GFRP & no preim & 72345 & 0,021 & 1 & 127 & 0,36 & anclado & $\mathrm{BH}$ & 9,74 & 900 & 0,0025 & 609,6 & 101,6 & 12 \\
\hline Galati & S\&-CO3 & & GFRP & no preim & 92900 & \begin{tabular}{|l|}
0,0182 \\
\end{tabular} & 1 & 76,2 & 0,36 & refuerzo & $\mathrm{BH}$ & 10,5 & 900 & 0,0025 & 600 & 92 & 13 \\
\hline$(2003)$ & S\&-CO5 & flexión & GFRP & no preim & 92900 & 0,0182 & 1 & 127,0 & 0,36 & & $\mathrm{BH}$ & 10,5 & 900 & 0,0025 & 600 & 92 & 13 \\
\hline & $\mathrm{S} \&-\mathrm{CO} 7$ & & GFRP & no preim & 92900 & \begin{tabular}{|l|}
0,0182 \\
\end{tabular} & 1 & 177,8 & 0,36 & anclar & $\mathrm{BH}$ & 10,5 & 900 & 0,0025 & 600 & 92 & 13 \\
\hline Galati & S19-CL3 & flexo- & GFRP & no preim & 92900 & 0,0182 & 1 & 76,2 & 0,36 & refuerzo & LP & 17,1 & 700 & 0,0035 & 600 & 64 & 18,8 \\
\hline$(2003)$ & S19-CL5 & compr. & GFRP & no preim & 92900 & 0,0182 & 1 & 127,0 & 0,36 & sin anclar & LP & 17,1 & 700 & 0,0035 & 600 & 64 & 18,8 \\
\hline \begin{tabular}{|l|} 
Ensayos \\
\end{tabular} & F.1.10_5 & & CFRP & no preim & 240000 & 0,0155 & 1 & 110 & 0,117 & & LM & 13,7 & 750 & 0,0035 & 235 & 110 & 9,4 \\
\hline Tesis & L.1.5_5 & flexo- & CFRP & preconf. & 150000 & 0,016 & 1 & 50 & 1,4 & & LM & 13,7 & 750 & 0,0035 & 235 & 110 & 9,2 \\
\hline & $\begin{array}{l}\text { L.2.5 } 5 \\
\end{array}$ & & CFRP & preconf. & 150000 & 0,016 & 1 & 100 & 1,4 & & LM & 13,7 & 750 & 0,0035 & 235 & 110 & 9,4 \\
\hline Triantafillou & $0-2 a$ & & CFRP & preconf. & 150000 & 0,014 & 1 & 100 & 1 & & LP & 3,0 & 1267 & 0,0035 & 400 & 120 & 7,5 \\
\hline (1998) & $0-2 b$ & flexión & CFRP & preconf. & 150000 & 0,014 & 1 & 100 & 1 & refuerzo & LP & 3,0 & 1267 & 0,0035 & 400 & 120 & 7,5 \\
\hline & $0-4 a$ & simple & CFRP & preconf. & 150000 & 0,014 & 1 & 200 & 1 & anclado & LP & 3,0 & 1267 & 0,0035 & 400 & 120 & 7,5 \\
\hline & $0-4 b$ & & CFRP & preconf. & 150000 & 0,014 & 1 & 200 & 1 & & LP & 3,0 & 1267 & 0,0035 & 400 & 120 & 7,5 \\
\hline Mosallam & WC-RET-O2 & & CFRP & no preim & 103400 & 0,0125 & 2 & 2640 & 0,584 & & LM & 25,0 & 771 & 0,0035 & 2640 & 101,6 & 26 \\
\hline$(2007)$ & WC-RE-09o & $\begin{array}{l}\text { flexion } \\
\text { simple }\end{array}$ & CFRP & no preim & 103400 & \begin{tabular}{|l|}
0,0125 \\
\end{tabular} & 1 & 2640 & 0,584 & refuerzo & LM & 25,0 & 771 & 0,0035 & 2640 & 101,6 & 26 \\
\hline & WE-RET-O2 & & GFRP & no preim & 18470 & \begin{tabular}{|l|l|}
0,022 \\
\end{tabular} & 3 & 2640 & 1,143 & anclado & LM & 25,0 & 771 & 0,0035 & 2640 & 101,6 & 26 \\
\hline & GGU & & GFRP & no preim & 70000 & 0,032 & 1 & 1000 & 0,67 & \begin{tabular}{|l|} 
sin anclar \\
\end{tabular} & LM & 8,2 & 739 & 0,0035 & 1000 & 110 & 9,1 \\
\hline & GBOU & & GFRP & no preim & 70000 & 0,032 & 1 & 1000 & 0,67 & \begin{tabular}{|l|} 
refuerzo \\
\end{tabular} & LM & 8,2 & 739 & 0,0035 & 1000 & 110 & 9,1 \\
\hline & GBAU & flexión & GFRP & no preim & 70000 & 0,032 & 1 & 1000 & 0,67 & anclado & LM & 8,2 & 739 & 0,0035 & 1000 & 110 & 9,1 \\
\hline & CGU & simple & CFRP & no preim & 230000 & 0,015 & 1 & 1000 & 0,165 & \begin{tabular}{|l|} 
sin anclar \\
\end{tabular} & LM & 8,2 & 739 & 0,0035 & 1000 & 110 & 9,1 \\
\hline & CBOU & bidir & CFRP & no preim & 230000 & 0,015 & 1 & 1000 & 0,165 & \begin{tabular}{|c|} 
refuerzo \\
\end{tabular} & LM & 8,2 & 739 & 0,0035 & 1000 & 110 & 9,1 \\
\hline & CBAU & & CFRP & no preim & 230000 & 0,015 & 1 & 1000 & 0,165 & anclado & LM & 8,2 & 739 & 0,0035 & 1000 & 110 & 9,1 \\
\hline & WRM2 & & GFRP & bidir. no pre & \begin{tabular}{|l|}
10520 \\
\end{tabular} & 0,0125 & 2 & 1000 & 1,2 & \begin{tabular}{|l} 
sin anclar \\
\end{tabular} & LM & 8,2 & 739 & 0,0035 & 1000 & 120 & 8,3 \\
\hline Barbieri & M10GtsT & flexo- & GFRP & no preim & 26130 & 0,022 & 1 & 250 & 1 & \begin{tabular}{|c|} 
refuerzo \\
\end{tabular} & LM & 9,8 & 462 & 0,0035 & 250 & 120 & 9,2 \\
\hline$(2000)$ & M1oCtsT & compr & CFRP & no preim & 230000 & 0,015 & 1 & 250 & 0,13 & anclado & LM & 9,8 & 462 & 0,0035 & 250 & 120 & 9,2 \\
\hline Galati & S12-CL5 & & GFRP & no preim & 92900 & 0,0182 & 1 & 127,0 & 0,36 & refuerzo & LP & 17,5 & 700 & 0,0035 & 600 & 95 & 12,6 \\
\hline & $\mathrm{S}_{12}-\mathrm{CO}_{3}$ & flexo- & GFRP & no preim & 92900 & 0,0182 & 1 & 76,2 & 0,36 & $\sin$ & $\mathrm{BH}$ & 10,5 & 900 & 0,0025 & 600 & 92 & 13,0 \\
\hline & S12-CO5 & & GFRP & no preim & 92900 & 0,0182 & 1 & 127,0 & 0,36 & anclar & $\mathrm{BH}$ & 10,5 & 900 & 0,0025 & 600 & 92 & 13,0 \\
\hline \begin{tabular}{|l|} 
Ensayos \\
\end{tabular} & F.3.10_5 & & CFRP & no preim & 240000 & \begin{tabular}{|l|}
0,0155 \\
\end{tabular} & 3 & 110 & 0,117 & & LM & 13,7 & 750 & 0,0035 & 235 & 110 & 9,2 \\
\hline Tesis & F.2.10 $\_3$ & flexo- & CFRP & no preim & 240000 & 0,0155 & 2 & 110 & 0,117 & & LM & 13,7 & 750 & 0,0035 & 235 & 110 & 9,3 \\
\hline & F.2.20 $\_3$ & & CFRP & no preim & 240000 & 0,0155 & 2 & 220 & 0,117 & & LM & 13,7 & 750 & 0,0035 & 235 & 110 & 9,1 \\
\hline \begin{tabular}{|l|} 
Albert \\
\end{tabular} & ICST-10 & flexión & CFRP & no preim & \begin{tabular}{|l|}
47375 \\
\end{tabular} & 0,013 & 1 & 250 & 0,73 & refuerzo & $\mathrm{BH}$ & 13,4 & 765 & 0,003 & 1205 & 193,4 & 20,9 \\
\hline$(1998)$ & ICST-12 & simple & CFRP & no preim & 30217 & 0,013 & 1 & 500 & 0,73 & & $\mathrm{BH}$ & 13,4 & 765 & 0,003 & 1205 & 193,4 & 20,9 \\
\hline Morbin & $\mathrm{COG}_{3}$ & & GFRP & no preim & 72345 & 0,021 & 1 & 76,2 & 0,36 & & $\mathrm{BH}$ & 9,74 & 900 & 0,0025 & 609,6 & 101,6 & 12 \\
\hline$(2002)$ & COG5 & & GFRP & no preim & 72345 & 0,021 & 1 & 127 & 0,36 & & $\mathrm{BH}$ & 9,74 & 900 & 0,0025 & 609,6 & 101,6 & 12 \\
\hline & COG7 & & GFRP & no preim & 72345 & 0,021 & 1 & 177,8 & 0,36 & & BH & 9,74 & 900 & 0,0025 & 609,6 & 101,6 & 12 \\
\hline & CLG3 & & GFRP & no preim & 72345 & 0,021 & 1 & 76,2 & 0,36 & & LP & 17,23 & 700 & 0,0035 & 609,6 & 101,6 & 12 \\
\hline & CLG5 & & GFRP & no preim & 72345 & 0,021 & 1 & 127 & 0,36 & refuerzo & LP & 17,23 & 700 & 0,0035 & 609,6 & 101,6 & 12 \\
\hline & CLG7 & flexión & GFRP & no preim & 72345 & 0,021 & 1 & 175 & 0,36 & & LP & 17,23 & 700 & 0,0035 & 609,6 & 101,6 & 12 \\
\hline & $\mathrm{COA}_{3}$ & simple & AFRP & no preim & 117130 & 0,017 & 1 & 76,2 & 0,28 & anclar & BH & 9,74 & 900 & 0,0025 & 609,6 & 101,6 & 12 \\
\hline & COA5 & & AFRP & no preim & 117130 & 0,017 & 1 & 127 & 0,28 & & $\mathrm{BH}$ & 9,74 & 900 & 0,0025 & 609,6 & 101,6 & 12 \\
\hline & CLA3 & & AFRP & no preim & 117130 & 0,017 & 1 & 76,2 & 0,28 & & LP & 17,23 & 700 & 0,0035 & 609,6 & 101,6 & 12 \\
\hline & CLA5 & & AFRP & no preim & 117130 & 0,017 & 1 & 127 & 0,28 & & LP & 17,23 & 700 & 0,0035 & 609,6 & 101,6 & 12 \\
\hline & CLA7 & & AFRP & no preim & 117130 & 0,017 & 1 & 177,8 & 0,28 & & LP & 17,23 & 700 & 0,0035 & 609,6 & 101,6 & 12 \\
\hline & CLA9 & & AFRP & no preim & 117130 & 0,017 & 1 & 228,6 & 0,28 & & LP & 17,23 & 700 & 0,0035 & 609,6 & 101,6 & 12 \\
\hline Hamilton & S1 & & GFRP & no preim & 11667 & 0,015 & 1 & 69,9 & 2 & & $\mathrm{BH}$ & 14,5 & 900 & 0,003 & 610 & 200 & 9,0 \\
\hline$(2001)$ & S2 & & GFRP & no preim & 11667 & 0,015 & 1 & 69,9 & 2 & & $\mathrm{BH}$ & 14,5 & 900 & 0,003 & 610 & 200 & 9,0 \\
\hline & S3 & flexión & GFRP & no preim & 11667 & 0,015 & 1 & 69,9 & 2 & refuerzo & $\mathrm{BH}$ & 10,9 & 900 & 0,003 & 610 & 200 & 9,0 \\
\hline & S4 & simple & GFRP & no preim & 11667 & 0,015 & 1 & 69,9 & 2 & anclado & BH & 10,9 & 900 & 0,003 & 610 & 200 & 9,0 \\
\hline & T1 & & GFRP & no preim & 11667 & 0,015 & 2 & 139,8 & 2 & & $\mathrm{BH}$ & 13,7 & 900 & 0,003 & 1220 & 200 & 23,5 \\
\hline & T2 & & GFRP & no preim & 11667 & 0,015 & 1 & 279,6 & 2 & & $\mathrm{BH}$ & 15,0 & 900 & 0,003 & 1220 & 200 & 23,5 \\
\hline Tan & WRM1 & Fl.sim.bi. & GFRP & bidir. no pre & 10520 & 0,0125 & 1 & 1000 & 1,2 & sin anclar & LM & 8,2 & 739 & 0,0035 & 1000 & 110 & 9,1 \\
\hline Barbieri & M10ClsT & fl-comp & CFRP & preconf. & 165000 & \begin{tabular}{|l|}
0,017 \\
\end{tabular} & 1 & 50 & 1,2 & anclado & LM & 9,8 & 462 & 0,0035 & 250 & 120 & 9,2 \\
\hline
\end{tabular}

AFRP: FRP con fibra de aramida, GFRP: FRP con fibra de vidrio, CFRP: FRP con fibra de carbono

no preim: hojas sólo de fibras unidireccionales no preimpregnadas

preconf.: bandas pultrusionadas preconformadas

bidir no pre: hojas sólo de fibras bidireccionales no preimpregnadas

LM: ladrillo macizo, LP: ladrillo perforado; BH: bloque de hormigón, h/t: esbeltez probeta 


\subsection{Revisión del método sin aplicar coeficientes de seguridad}

En primer lugar, se comprueba si la predicción teórica obtenida con el método propuesto tanto del modo de fallo como del momento último se aproxima a los resultados publicados. En esta tarea se utilizan exclusivamente los datos facilitados por cada autor, incluida la deformación máxima alcanzada por la lámina durante el ensayo que se utiliza como valor de deformación de cálculo del refuerzo. La comprobación sólo puede hacerse para 46 ensayos de la base (no puede hacerse para las pruebas donde no se conoce la deformación máxima experimentada por el refuerzo siendo el fallo atribuible a él).

Se comprueba que en cerca del $90 \%$ de los casos se predijo correctamente el modo de fallo y que el promedio del cociente momento último experimental entre el momento último teórico está relativamente próximo a la unidad (se obtiene un promedio 0,87 con una desviación estándar de 0,19). Aunque el ajuste es razonable, la mayoría de los resultados experimentales están algo por debajo de la predicción teórica, por lo que la predicción resulta insegura en tanto no se apliquen coeficientes de seguridad. En la Figura 5 se muestran gráficamente los resultados obtenidos.

\subsection{Determinación del coeficiente reductor por adherencia y revisión del método incorporando la seguridad de los materiales}

A continuación se busca una buena estimación de la deformación efectiva de cálculo del refuerzo a efectos de adherencia. Se tantean dos posibles aproximaciones para determinar su valor, ambas basadas en las propuestas de dos guías técnicas elaboradas por el ACI (4) (19). En esta tarea se utilizan los 46 ensayos de la base de datos cuyo fallo es atribuible al refuerzo. Ambas aproximaciones son de aplicación para refuerzos con los extremos anclados porque aproximadamente la mitad de las probetas de la base tienen los extremos de la lámina fijados mecánicamente.

Hay que señalar que la base de datos tiene una extensión limitada por lo que resulta insuficiente para determinar un coeficiente reductor con el que obtener valores que puedan ser considerados «de cálculo» con el nivel de fiabilidad habitualmente admitido. No obstante, el trabajo realizado que se expone a continuación sí que permite proponer y validar un coeficiente reductor en línea con los propuestos por otros investigadores.

En primer lugar se plantea que la deformación que como máximo puede admitir la lámina de FRP para no presentar problemas de despegue en la zona interior del elemento sea una fracción de la deformación última del material facilitada por el fabricante, tal y como plantea la guía ACI $440.7 \mathrm{R}-10$, según la ecuación [16]. Qué fracción es la adecuada quedaría cuantificado por una constante que se ha denominado $K_{1}$. El valor característico que toma esta constante $K_{1}$ calculada para cada uno de los 46 ensayos analizados es igual a o,43. En la Figura 6 se representa el valor obtenido para cada ensayo.

$$
\varepsilon_{f e}=K_{1} \varepsilon_{f u}^{*}
$$

La mayor parte de los refuerzos de los ensayos son tejidos flexibles de fibra de vidrio no preconformados. Menos datos

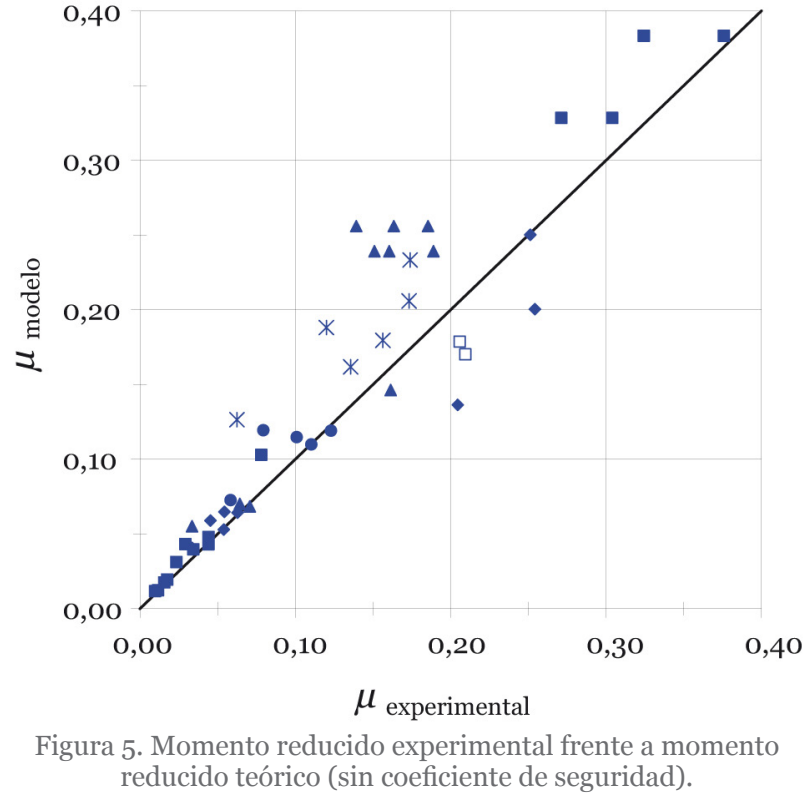

se tienen de tejidos no preconformados de aramida y carbono. Aún con ello, los resultados de estos tres tipos de refuerzo son relativamente homogéneos y permiten proponer un valor de $K_{1}$ igual a 0,40 . Los valores más bajos de esta constante se obtienen para los refuerzos preconformados de fibra de carbono. A pesar de las pocas muestras con este tipo de refuerzo, parece razonable adoptar para ellos un valor de $K$ inferior, por ejemplo, 0,25 (el valor característico sólo para las bandas preconformadas es de 0,26 ).

En segundo lugar se plantea que la deformación máxima que la lámina de FRP puede desarrollar por adherencia sea directamente proporcional a la raíz de la resistencia a compresión del soporte e inversamente proporcional a la raíz del producto del número de capas de refuerzo por su rigidez y su espesor, tal y como plantea la guía ACI 440.2R-o8, según la ecuación [17]. El valor característico que toma la constante $K_{2}$ calculada para cada uno de los 46 ensayos es igual a 0,36 . En la Figura 7 se representa el valor de la constante obtenido para cada ensayo. Como la rigidez del refuerzo interviene en el cálculo de esta constante, el valor propuesto, $K_{2}=0,25$, parece válido para todos formatos.

$$
\varepsilon_{f e}=K_{2} \sqrt{\frac{f_{m k}}{n E_{f} t_{f}}}
$$

Por último se revisa el modelo incorporando la seguridad de los materiales: para la fábrica se utiliza un coeficiente de seguridad igual a 2,5 y para la lámina se emplean los valores de deformación de cálculo antes propuestos (lo que equivale a utilizar una resistencia minorada del material). No se aplican coeficientes reductores medioambientales porque los ensayos se realizan en condiciones protegidas. Se hacen con un criterio común respecto al módulo elasticidad de la fábrica, igual a 750 veces su resistencia de cálculo, y a la deformación última de la fábrica o,0035 (EC-6).

Si la comprobación se hace con la constante $K_{2}$ se obtienen buenos resultados y la predicción es segura para el $94 \%$ de las pruebas. Pero cuando se hace con la constante $K_{1}$ el resultado es aún mejor (98,5 \% de resultados seguros). En la 
Figura 8 se muestra gráficamente los resultados obtenidos cuando se emplea la constante $K_{1}$.

A pesar de no haber diferencias sustanciales (con ambas propuestas se ha obtenido un buen resultado), la primera aproximación es más sencilla y se ajusta algo mejor a los resultados experimentales por lo que se propone calcular la deformación de cálculo del refuerzo como el producto de una constante igual a 0,40 (para refuerzos ejecutados in situ) o 0,25 (para laminados preconformados de fibra de carbono) por la deformación última de la lámina. Este coeficiente se ha podido calibrar con un mayor número de pruebas que la propuesta para refuerzos exteriores incluida en la guía ACI 440-7R-10 donde se utilizaron un total de 27 ensayos, todos ellos de compresión simple, procedentes de tres únicas campañas experimentales (21).

\section{DIAGRAMAS DE INTERACCIÓN ADIMENSIONALES AXIL-MOMENTO}

Con las ecuaciones de [6] a [15] se pueden elaborar gráficos de interacción adimensionales axil-momento para distintas cuantías mecánicas de refuerzo $\left(\omega_{f}\right)$ como los de las Figuras 9 y 10. Estos dos gráficos se han preparado para el supuesto de que la deformación efectiva de cálculo del refuerzo $\left(\varepsilon_{f e}\right)$ sea igual a 0,006 y 0,0035, valores adecuados para dos posibles sistemas de refuerzo con FRP existentes en el mercado: un refuerzo con hoja flexible unidireccional de fibra de vidrio ejecutada in situ con $\varepsilon_{f u}{ }^{*}=0,02$ situada al interior de un edificio y una banda preconformada de fibra de carbono $\operatorname{con} \varepsilon_{f u}{ }^{*}=0,017$ situada al exterior. Además con ayuda de las ecuaciones para la determinación de la cuantía mecánica elástica $\left(\omega_{f, \text { elást }}\right)$ y cuantía mecánica límite $\left(\omega_{f, \text { lim }}\right)$ según las ecuaciones [6] y [7] respectivamente, se pueden visualizar qué zonas del gráfico se corresponden con cada uno de los posibles dominios de deformación en la rotura, y por tanto conocer el modo de fallo esperable para una fábrica y sistema de refuerzo determinados.

Los gráficos de interacción axil-momento no son sólo una herramienta que facilita la labor de diseño y comprobación de la sección reforzada sino que además permiten analizar la incidencia de algunas de las variables involucradas en la capacidad resistente a flexión de la sección, como por ejemplo el axil.

Como puede observarse en las Figuras 9 y 10, cuando el axil es bajo la mejora que se obtiene con la adición del refuerzo es notable, de ahí que en la bibliografía publicada sobre el tema estén documentadas grandes mejoras cuando las probetas de fábrica reforzadas se ensayan a flexión simple. En este rango de compresión baja o moderada, hasta aproximadamente el $30 \%$ de la carga última del muro en compresión centrada, predominan los dominios de deformación de tipo AI y AII (fallo atribuible al refuerzo). En estos dominios, las curvas representativas de la capacidad resistente de la sección para distintas cuantías mecánicas de refuerzo son prácticamente paralelas lo que significa que cuando la cuantía mecánica se incrementa en una determinada cantidad se consigue un incremento del momento similar tanto si se parte de una cuantía pequeña como grande.

Sin embargo, para niveles de compresión elevados (por encima del $50 \%$ de la carga última del muro) predomina el dominio de deformación de tipo B (fallo por compresión de la

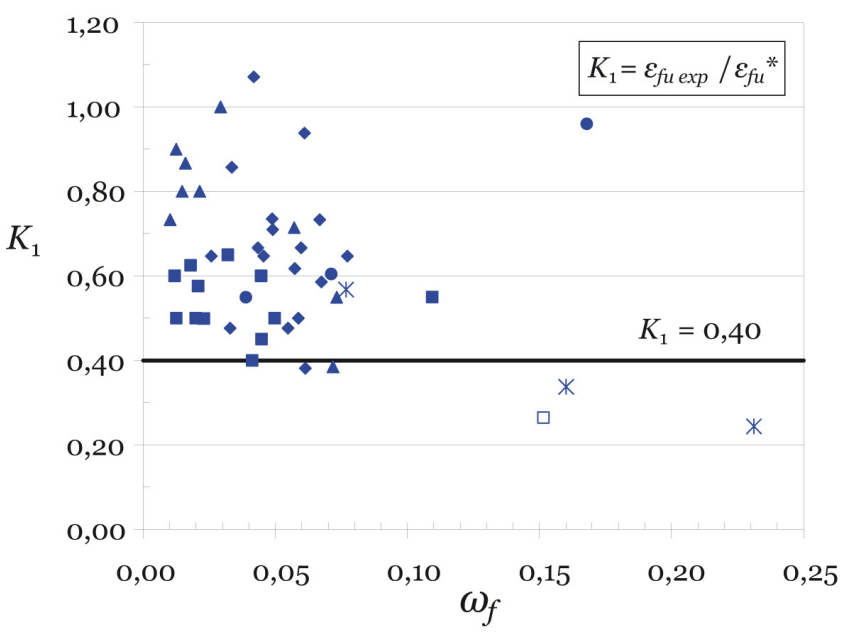

Figura 6. Valor de la constante $K_{1}$ en los ensayos de la base de datos con fallo atribuible al refuerzo.

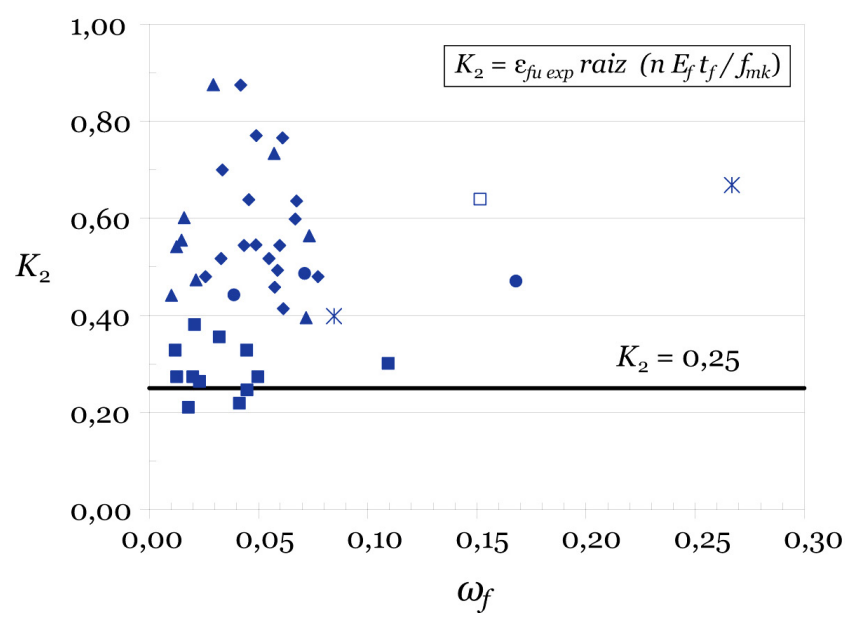

Figura 7. Valor de la constante $K_{2}$ en los ensayos de la base de datos con fallo atribuible al refuerzo.

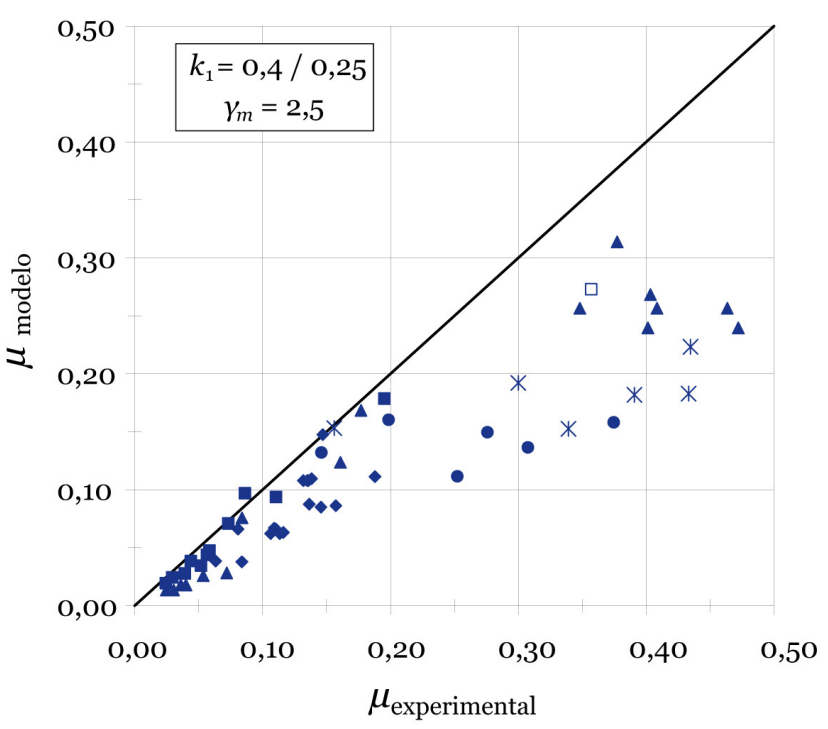

Figura 8. Momento reducido experimental frente a momento reducido teórico (incorporando la seguridad de los materiales). 


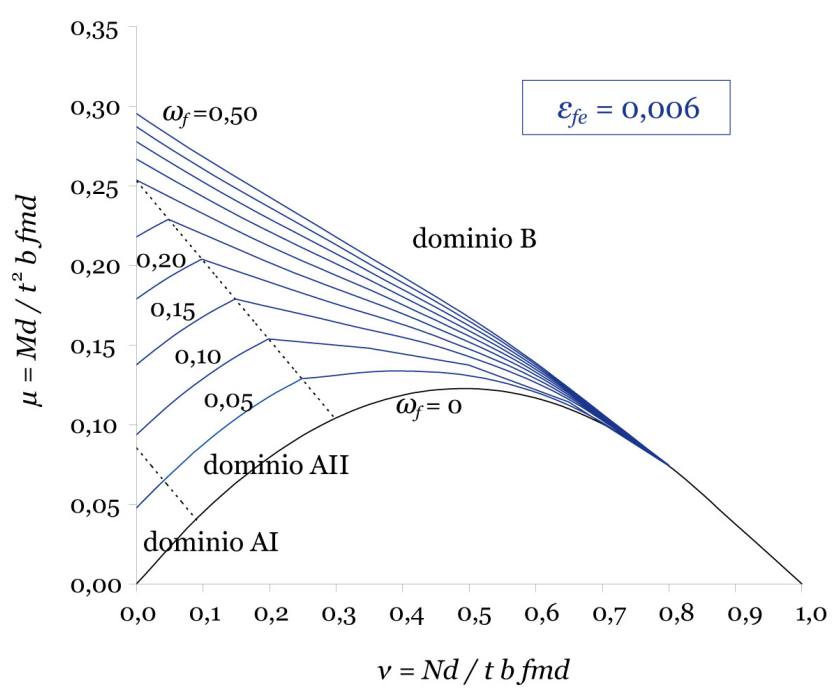

Figura 9. Diagrama adimensional axil -momento para distintas cuantías mecánicas de refuerzo considerando un $\varepsilon_{f e}=0,006$ con $\varepsilon_{m u}=0,0035 \mathrm{y} \beta=750$.

fábrica) y el incremento de momento obtenido con el refuerzo es cada vez menor. De hecho, las curvas representativas de la capacidad resistente de la sección de fábrica para distintas cuantías de refuerzo prácticamente se superponen entorno a un 70-80 \% de la compresión última.

Con carácter general y hasta entorno a la mitad de la compresión máxima que resiste en compresión centrada, el problema de un muro de fábrica no armada es de estabilidad y no de resistencia. Es en este rango de compresiones no elevadas donde la aplicación del refuerzo exterior con materiales compuestos tiene sentido: aporta a la fábrica una capacidad para resistir tracciones que ésta no tiene y dicha capacidad permite equilibrar momentos solicitantes mayores. En cambio, para niveles de compresión elevados la incorporación del refuerzo apenas supone mejora.

Lo habitual es que las estructuras de fábrica se encuentren en un rango de compresiones bajas o moderadas, de no ser así, no tiene sentido proceder al refuerzo a flexión con materiales compuestos avanzados.

\section{RESUMEN Y CONCLUSIONES}

En este trabajo se presenta un método de comprobación en agotamiento de secciones de fábrica reforzadas exteriormente con materiales compuestos avanzados para solicitaciones combinadas de flexión y compresión. Está basado en el método de cálculo de la capacidad resistente de secciones de hormigón armado ajustando las variables específicas de aplicación en las fábricas reforzadas a partir de los resultados de una base de datos con ensayos de flexión y flexocompresión procedentes de un trabajo experimental propio y de otros nueve trabajos publicados. En el trabajo se muestra el procedimiento seguido para procesar toda esta información experimental lo que permite revisar el método y ajustar las variables específicas para las fábricas.

En concreto, se propone emplear un «coeficiente reductor por adherencia» para prevenir el fallo prematuro debido al desprendimiento del refuerzo inducido por la apertura de

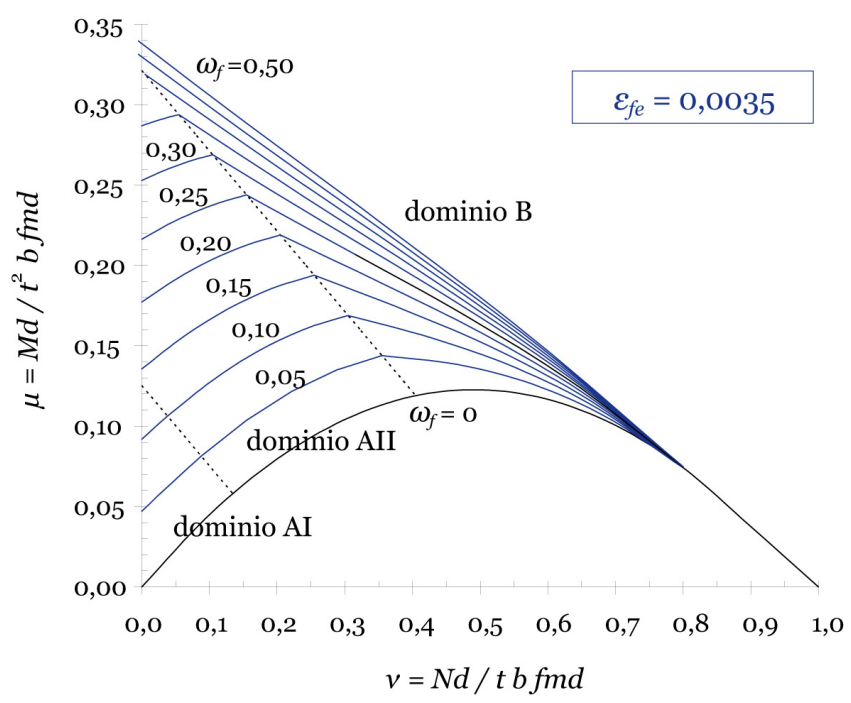

Figura 10. Diagrama adimensional axil -momento para distintas cuantías mecánicas de refuerzo considerando un $\varepsilon_{f e}=0,0035$ con $\varepsilon_{m u}=0,0035$ y $\beta=750$.

fisuras. Tras analizar dos posibles aproximaciones para estimarlo, se propone que se calcule como una fracción de la deformación última del material (tal y como plantea la guía ACI 440.7R-10). Dicha fracción queda fijada mediante un factor $K$ igual a o,40 para láminas de hojas flexibles sólo de fibras donde el material compuesto se ejecuta in situ y 0,25 para bandas preconformadas de fibra de carbono. Esta propuesta debe emplearse para refuerzos con los extremos anclados.

Se comprueba que el método propuesto permite estimar el momento último de secciones de fábrica reforzadas con láminas de FRP cuando se utiliza: 1- una deformación de cálculo del refuerzo calculada con el coeficiente reductor por adherencia $K$ indicado anteriormente; 2- un coeficiente de seguridad parcial de la fábrica igual a 2,5; 3- un módulo elástico de la fábrica igual a 750 veces su resistencia de cálculo y 4- una deformación última de la fábrica igual a 0,0035 (EC-6). Con estas condiciones, el método ha resultado seguro para el 98,5\% de las pruebas de la base de datos.

El nivel de compresión soportado por el muro es determinante y afecta a la mejora en la capacidad resistente a flexión conseguida con el refuerzo. Cuando esta compresión es baja o moderada, hasta aproximadamente el 30 \% de la carga última del muro en compresión centrada, la incorporación del refuerzo proporciona importantes mejoras. En cambio, para niveles de compresión elevados (por encima del $50 \%$ de la compresión última) la incorporación del refuerzo apenas supone mejora. Lo habitual es que las estructuras de fábrica se encuentren en un rango de compresiones bajas o moderadas, de no ser así, no tiene sentido proceder al refuerzo a flexión con materiales compuestos avanzados.

\section{AGRADECIMIENTOS}

Este trabajo se ha desarrollado gracias a una beca predoctoral concedida por la Universidad Politécnica de Madrid y la colaboración del Instituto de Ciencias de la Construcción Eduardo Torroja. 


\section{REFERENCIAS}

(1) Bakis, C., Bank, L., Brown, V., Cosenza, E., Davalos, J., Lesko, J., Rizcalla, S., Triantafillou, T. (2002). Fiber-Reinforced Polymer Composites for Construction - State-of-the-Art Review. Journal of Composites for Construction, 6(2): 73-87, doi: http://dx.doi.org/10.1061/(ASCE)1090-0268(2002)6:2(73).

(2) Celestini, G., Casadei, P. (2009, 22-24 Abril). Innovation on Advanced Composite Materials for Strengthening and Protection of Historical Masonry Structures. En Proceedings of Meccanica delle strutture in muratura rinforzate con compositi: modellazione, sperimentazione, progetto, controllo (pp. 184-191). Venezia: Edición de C. Gentilini.

(3) Credali, L. (2009, 22-24 Abril). L'impiego delle tecnologie FRP nel recupero strutturale di Edifici Storici. En Proceedings of Meccanica delle strutture in muratura rinforzate con compositi: modellazione, sperimentazione, progetto, controllo (pp. 208-223). Venezia: Edición de C. Gentilini.

(4) ACI. (2010). 440.7R-10 Guide for Design and Construction of Externally Bonded FRP Systems for Strengthening Unreinforced Masonry Structures. American Concrete Institute (ACI).

(5) CNR. (2004). CNR-DT 200/2004 - Guide for the Design and Construction of Externally Bonded FRP Systems for Strengthening Existing Structures. Rome: Consiglio Nazionalle delle Ricerche (CNR).

(6) CNR. (2012). CNR-DT 200 R1/2012 - Istruzioni per la Progettazione, l'Esecuzione ed il Controllo di Interventi di Consolidamento Statico mediante l'utilizzo di Compositi Fibrorinforzati. Rome: Consiglio Nazionalle delle Ricerche (CNR).

(7) Albert, M.L., Cheng, R.J.J., Elwy, E.E. (1998). Rehabilitation of Unreinforced Masonry Walls with Externally applied Fiber Reinforced Polymers. Structural Engineering Report $n^{0}{ }^{2} 26$. Alberta: Dep. of Civil \& Environmental Engineering of University of Alberta.

(8) Velázquez-Dimas, J.I., Ehsani, M.R. (2000). Modeling Out-of-plane Behavior of URM Walls Retrofitted with Fiber Composites. Journal of Composites for Construction, 4(4): 172-181, doi: http://dx.doi.org/10.1061/(ASCE)10900268(2000)4:4(172).

(9) Hamilton III, H.R., Dolan, C.W. (2001). Flexural Capacity of Glass FRP Strengthened Concrete Masonry Walls. Journal of Composites for Construction, 5(3): 170-178, doi: http://dx.doi.org/10.1061/(ASCE)1090-0268(2001)5:3(170).

(10) Morbin, A. (2002). Strengthening Masonry Elements with FRP Composites. Structural Report CIES 02-23. Center for Infrastructure Engineering Studies (CIES). Rolla: University of Missouri-Rolla.

(11) Tan, K.H., Patoary, M.H. (2004). Strengthening of Masonry Walls Against Out-of-plane Loads Using Fiber-Reinforced Polymer Reinforcement. Journal of Composites for Construction, 8(1): 79-87, doi: http://dx.doi.org/10.1061/ (ASCE)1090-0268(2004)8:1(79).

(12) Mosallam, A.S. (2007). Out-of-plane Flexural Behavior of Unreinforced Red Brick Walls Strengthened with FRP Composites. Composites. Part B: Engineering, 38(5-6): 559-574, doi: http://dx.doi.org/10.1016/j.compositesb.2006.07.019.

(13) Barbieri, A. (2000). Interventi Strutturali su Edifici Storici in Muratura: Elementi Pressoinflessi, Rinforzati con Materiale Composito (Tesis Doctoral). Lecce: University of Lecce.

(14) Galati, N., Tumialan, G., Nanni, A. (2003). Arching Effect in Masonry Walls Reinforced with Fiber Reinforced Polymer (FRP) Materials. Structural Report CIES 03-33, Center for Infrastructure Engineering Studies (CIES). Rolla: University of Missouri-Rolla.

(15) Accardi, M., Cucchiara, C., Faila, A., La Mendola, L. (2007, 16-18 Julio). CFRP flexural strengthening of masonry walls: experimental and analytical approach. En Proceedings of FRPRCS-8 Conference, CD - ${ }^{\circ}$ paper: 347 . Patras (Greece): University of Patras.

(16) Martínez, S. (2013). Comportamiento estructural de elementos de fábrica reforzados con materiales compuestos avanzados solicitados a flexión y flexocompresión (Tesis Doctoral). Madrid: Escuela Técnica Superior de Arquitectura de Madrid.

(17) Triantafillou, T.C. (1998). Strengthening of Masonry Using Epoxy-Bonded FRP Laminates. Journal of Composites for Construction, 2(2): 96-104, doi: http://dx.doi.org/10.1061/(ASCE)1090-0268(1998)2:2(96).

(18) Oller, E., Mari, A.R., Bellido, L. (2014). Dimensionamiento del refuerzo a flexión con laminados de polímeros reforzados con fibras (FRP) evitando su desprendimiento prematuro. Informes de la Construcción, 65(532), 519-531, doi: http:// dx.doi.org/10.3989/ic.12.087.

(19) ACI. (2008). 440.2R-o8 Guide for Design and Construction of Externally Bonded FRP Systems for Strengthening Concrete Structures. American Concrete Institute (ACI).

(20) Hendry, A.W. (1998). Masonry materials in compression. En Structural Masonry (Second Edition), pp. 13-51. Macmillan Press Ltd.

(21) Tumialan, J.G., Gold, W.J., Galati, N., Prota, A. (2011). ACI Design Guide for Flexural and Shear Strengthening of URM Walls with FRP Systems. ACI Special Publication, 275. 